\title{
Transverse Digital Damper System for the Fermilab Anti-Proton Recycler
}

\author{
N. Eddy and J. Crisp \\ Fermi National Accelerator Laboratory, P.O. Box 500, Batavia, IL 60510
}

\begin{abstract}
A transverse damping system is used in the Recycler at Fermilab to damp beam instabilities which arise from large beam intensities with electron cooling. Initial tests of electron cooling demonstrated beam loss due to transverse beam motion when the beam was cooled past the beam density threshold. The transverse damper system consists of two horizontal and two vertical pickups whose signals are amplified and passed into an analog hybrid to generate a difference signal from each pickup. The difference signals are input to a custom digital damper board which digitizes the analog signals at $212 \mathrm{mhz}$, performs digital processing of the signals inside a large Altera Stratix II FPGA, then provides analog output at $212 \mathrm{mhz}$ via digital to analog converters. The digital damper output is sent to amplifiers which drive one horizontal and one vertical kicker. An initial prototype digital damper board has been successfully used in the Recycler for over six months. Currently, work is underway to replace the prototype board with an upgraded VME version.
\end{abstract}

\section{INTRODUCTION}

The Recycler at Fermilab is used to store and cool anti-protons. In the last year, electron cooling has been used to cool the anti-protons to increase anti-proton density and improve the Tevatron luminosity. The increased beam density gives rise to transverse instabilities which can lead to emittance blowup and loss of beam [1]. A transverse damper which provides active beam feedback is used to keep the beam stable [2]. Figure 1 shows a simplified diagram of the system. The feedback system consists of a beam pickup, a filter to reject revolution harmonics, a delay line, power amplifiers, and a kicker. Transverse damping is accomplished by two independent systems acting in the horizontal and vertical planes.

\section{DAMPER FEEDBACK SYSTEM}

The beam pickups are split tube bpm pickups. Two pickups in each plane approximately $90^{\circ}$ apart in betatron phase are combined with a ratio to simulate the optimum betatron phase advance. The signals from the pickups are driven over several hundred feet of cable by pre-amplifiers in the tunnel. Signals from each pickup are input to an analog hybrid module which outputs a sum and difference signal. 


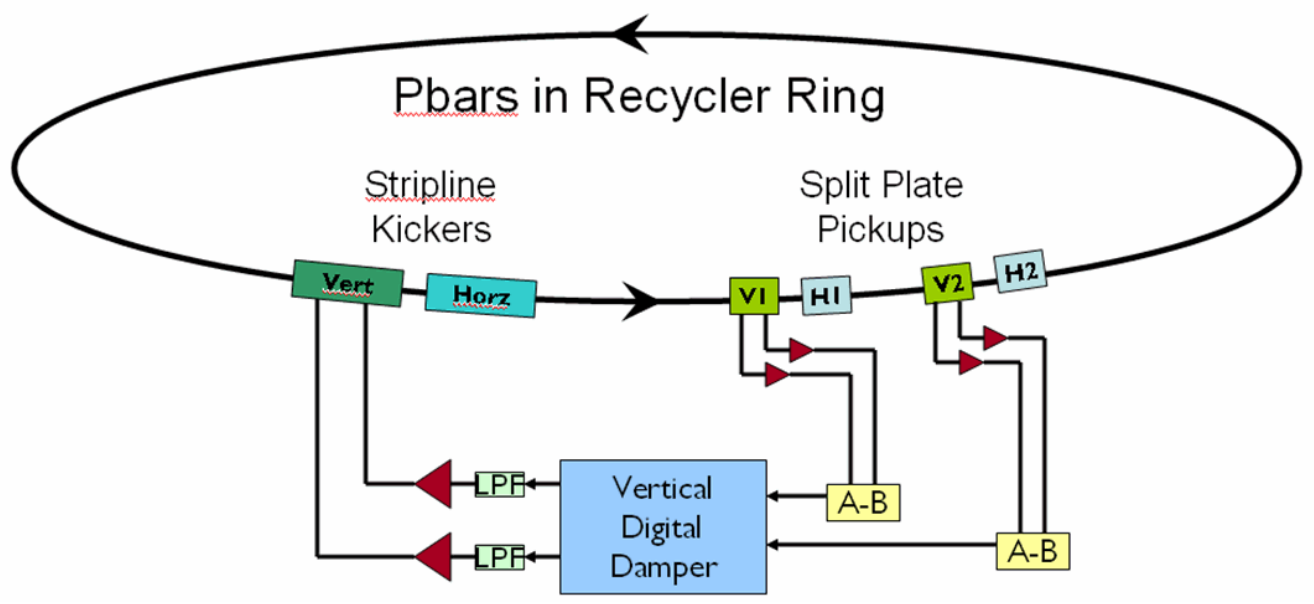

FIGURE 1. Overview of the Recycler Transverse Damper system. The signal path for the vertical damper is shown with the horizontal damper being similar.

The difference signals from the hybrid are input to a custom board which digitizes the signals and processes them. The board uses a large fpga to implement the filtering, gain, and delays digitally. Fast digital to analog converters provide the drive signal for the beam kickers.

The feedback signal is driven to $1.4 \mathrm{~m}$ long stripline kickers by Amplifier Research KMA2020 100watt amplifiers. The amplifiers provide $53 \mathrm{db}$ of gain and flat phase response from $10 \mathrm{KHz}$ to $250 \mathrm{MHz}$. The kickers have a linear phase but are $3 \mathrm{db}$ down at $50 \mathrm{MHz}$ and switch sign at $107 \mathrm{MHz}$.

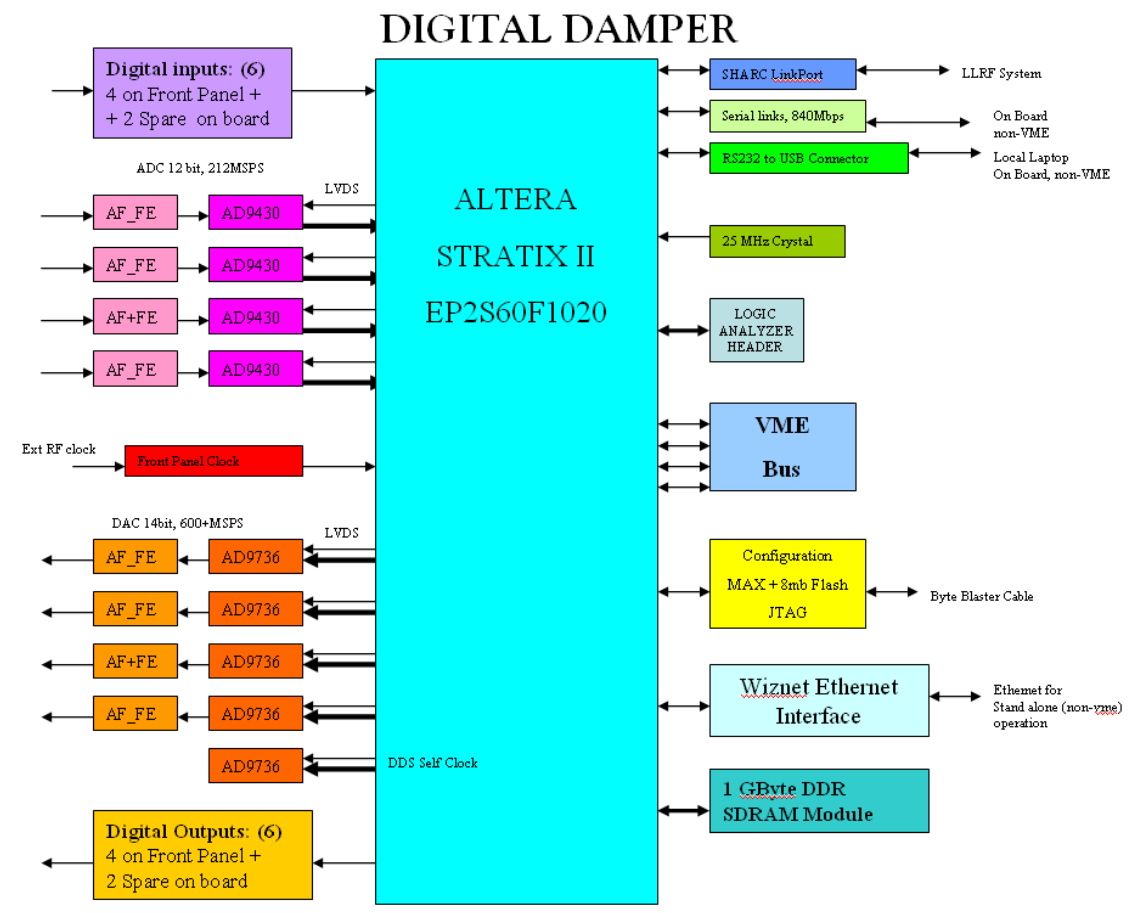

FIGURE 2. Block diagram of the custom digital damper board. 


\section{DIGITAL DAMPER}

The digital damper is a custom designed board and is based upon a prototype board developed by G.W. Foster and A. Seminov [3]. Figure 2 displays the basic block diagram of the board. The board contains four 12 bit 212Msps AD9430 ADCs input to an fpga which drives four channels of 14 bit 212Msps AD9736 DACs. The fpga is an Altera Stratix II EP2S60. The fpga handles all signal processing and I/O on the board including the VME slave interface.

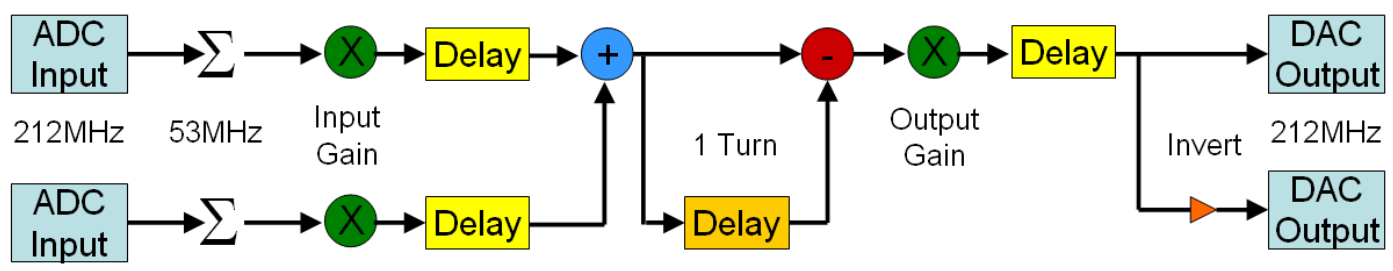

FIGURE 3. Digital filter, gain, and delay implemented in the fpga for the Recycler damper.

The algorithm for the Recycler damper is shown in Figure 3. Four successive adc inputs are summed to provide a 53MHz low pass filter and limit the output bandwidth. Programmable gains and delays in $212 \mathrm{MHz}$ clocks are used to combine the difference signals from two pickups to simulate an ideal betatron phase. The bpm inputs are combined as phase vectors to simulate the ideal bpm to kicker phase advance. A one turn notch filter is used to eliminate rotation harmonics from the beam signal. Programmable output gain is used to control the overal digital gain for the amount of beam present in the Recycler. The output can also be delayed up to one full turn in $53 \mathrm{MHz}$ clocks.

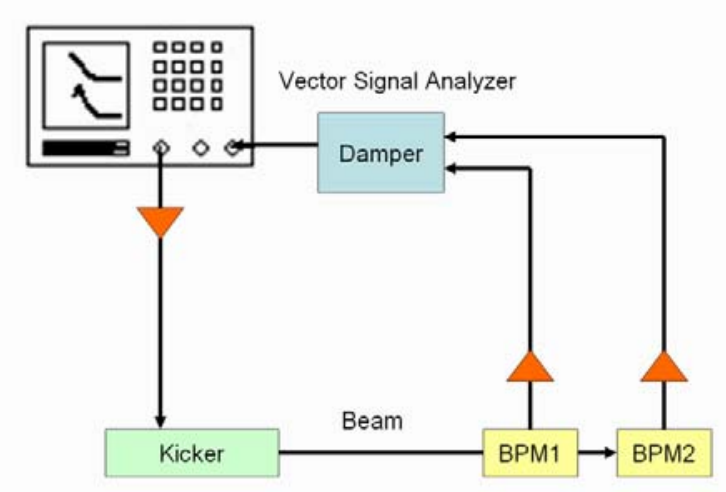

(a)

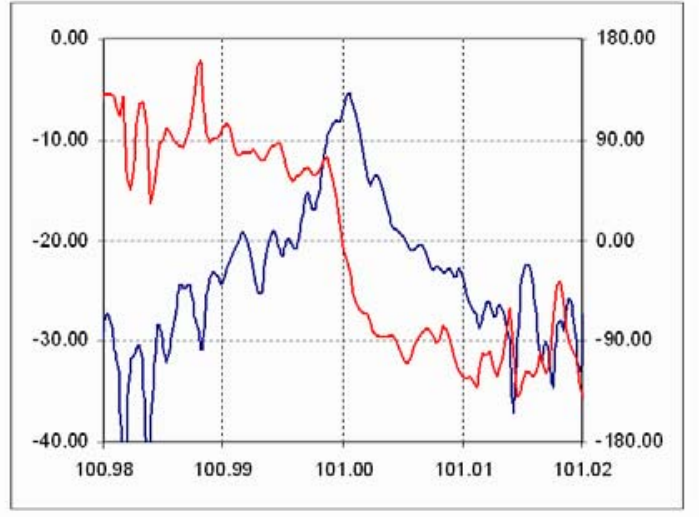

(b)

FIGURE 4. (a) The setup for the beam transfer function measurement. (b) An example beam transfer function measurement on the $101^{\text {st }}$ betatron harmonic - the inverted output is used so that the phase goes through zero. 


\section{TESTING AND RESULTS}

A vector signal analyzer is used to make beam transfer function (BTF) measurements of the betatron tune lines around the revolution harmonics. The measurement is made as shown in Figure 4a. For ideal damping, the phase should be $180^{\circ}$ when the amplitude is a maximum as shown in Figure $4 \mathrm{~b}$. Filter delay errors will show up as frequency dependent phase errors in the BTF measurements. If the input gains due to the lattice are not correct, it will produce a shift between the upper and lower tunes. This measurement is performed when fpga firmware is updated on the damper board to insure the filter delays are unchanged and also when the optics of the Recycler have changed.

To demonstrate that the damper was initially working, the beam was given a transverse kick to initiate oscillations. Figure 5a shows the result with the damper turned off. The oscillations have a decay time of about 400 turns due to chromaticity and landau damping. Figure $5 \mathrm{~b}$ shows the oscillations die away much quicker with the damper turned on. The oscillations have a decay time of about 150 turns with the damper on. Note, the transverse emittance preservation is improved with the damper on. The decay time is inversely proportional to the overall gain of the damper while the emittance growth of the beam due to system noise grows as the gain is increased. Tests have shown that if the digital gain is greater than 1, the system noise is dominated by the pre-amplifier noise figure. For less than unity digital gain, the system noise is dominated by adc noise.

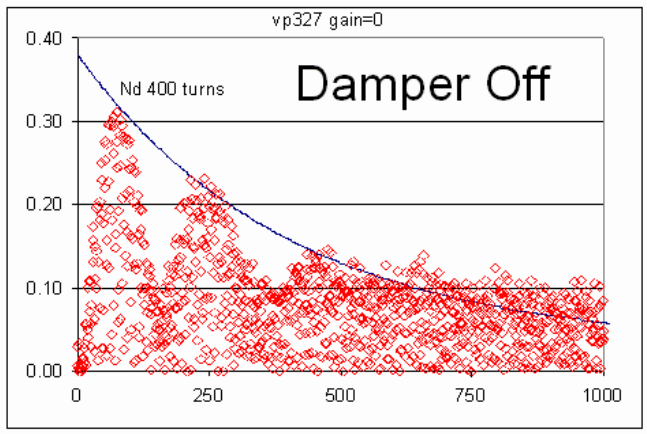

(a)

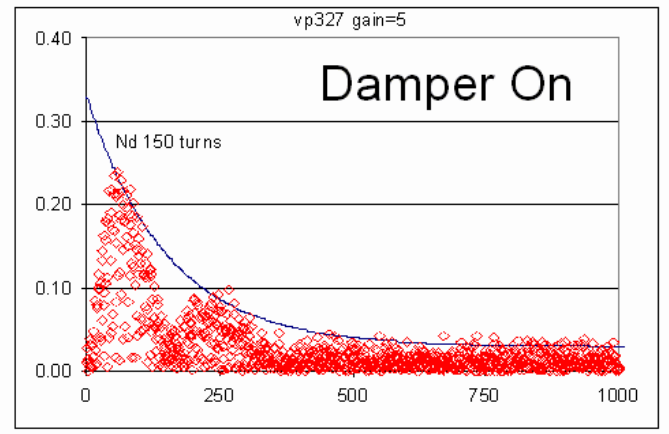

(b)

FIGURE 5. Beam oscillation amplitudes due to a transverse kick. (a) A natural damping time of 400 turns with the damper off. (b) The oscillations damped out in 150 turns with the damper on.

Transverse instabilities have been regularly observed in the Recycler before the damper was installed. Figure 6 shows bpm data taken during an indeuced transverse instability in DC proton beam. Figure 6a shows the growth of the oscillations until beam loss occurs. Figure $6 \mathrm{~b}$ shows beam oscillations shortly before the beam loss occurs. To first order, the beam stability threshold can be expressed in terms of beam density, D, defined as

$$
D=\frac{N}{\varepsilon_{L} \varepsilon_{T}}
$$


where $N$ is number of particles in e10, $\varepsilon_{L}$ is $95 \%$ longitudinal emittance in eVsec, and $\varepsilon_{T}$ is the $95 \%$ transverse emittance in $\pi \mathrm{mm} \mathrm{mR}$. Without the damper, transverse instabilities were observed when $\mathrm{D}$ was greater than 0.8 . Since installing the damper feedback system, the Recycler beam has remained stable with D as high as 1.6.

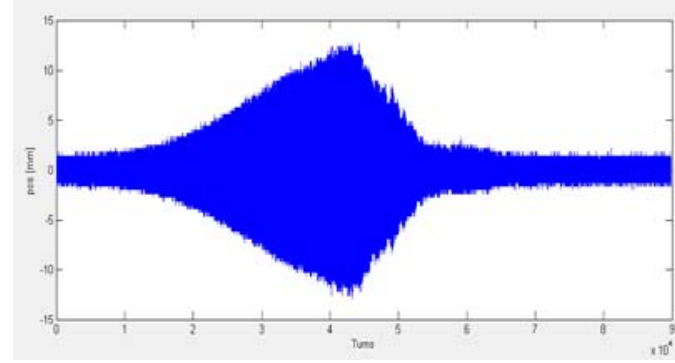

(a)

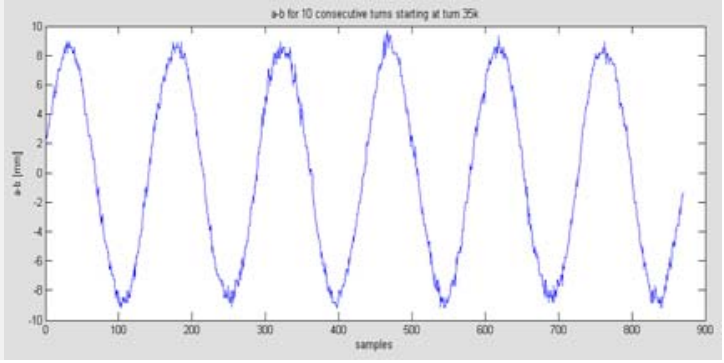

(b)

FIGURE 6. Transverse instability captured by bpm system. (a) The slow growth of the instability until beam loss occurs. (b) Beam oscillations just before beam loss.

\section{ACKNOWLEDGMENTS}

We would like to thank Martin Hu of the Recycler Department for his help in characterizing the transverse instabilities and providing support for the initial beam tests of the damper system.

\section{REFERENCES}

1. V. Balbekov, "Transverse coherent instability of a bunch in a rectangular potential well", FERMILAB-PUB06-070-AD, Apr 2006.

2. J. Crisp, M. Hu, V. Tupikov, "Fermilab Recycler Damper Requirements and Design", Particle Accelerator Conference, Knoxville, 2005.

3. G.W. Foster et al., "Main Injector/RR Damper”, Fermilab Beams Document 605v1 (2003). 